\title{
Clinically early-stage CSPa mutation carrier exhibits remarkable terminal stage neuronal pathology with minimal evidence of synaptic loss
}

\author{
Bruno A. Benitez ${ }^{1 *}$ D, Nigel J. Cairns ${ }^{2,3,6}$, Robert E. Schmidt ${ }^{3}$, John C. Morris², Joanne B. Norton ${ }^{4}$, \\ Carlos Cruchaga ${ }^{4,6}$ and Mark S. Sands ${ }^{1,5,6}$
}

\begin{abstract}
Autosomal dominant adult-onset neuronal ceroid lipofuscinosis (AD-ANCL) is a multisystem disease caused by mutations in the DNAJC5 gene. DNAJC5 encodes Cysteine String Protein-alpha (CSPa), a putative synaptic protein. AD-ANCL has been traditionally considered a lysosomal storage disease based on the intracellular accumulation of ceroid material. Here, we report for the first time the pathological findings of a patient in a clinically early stage of disease, which exhibits the typical neuronal intracellular ceroid accumulation and incipient neuroinflammation but no signs of brain atrophy, neurodegeneration or massive synaptic loss. Interestingly, we found minimal or no apparent reductions in CSPa or synaptophysin in the neuropil. In contrast, brain homogenates from terminal AD-ANCL patients exhibit significant reductions in SNARE-complex forming presynaptic protein levels, including a significant reduction in CSPa and SNAP-25. Frozen samples for the biochemical analyses of synaptic proteins were not available for the early stage AD-ANLC patient. These results suggest that the degeneration seen in the patients with AD-ANCL reported here might be a consequence of both the early effects of CSPa mutations at the cellular soma, most likely lysosome function, and subsequent neuronal loss and synaptic dysfunction.
\end{abstract}

Keywords: Neuronal ceroid lipofuscinosis, Cysteine string protein alpha, Synaptic loss, Neurodegenerative disease, Lysosome DNAJC5

\section{Background}

The neuronal ceroid lipofuscinoses (NCLs) are the most common group of inherited neurodegenerative diseases in children [1]. NCLs are a genetically, clinically and pathologically heterogeneous group of disorders, unified by the presence of intracellular accumulation of autofluorescent ceroid-lipofuscin aggregates (a complex mixture of proteins, lipids and metals) [1,2]. The age at onset of clinical symptoms and differences in ultrastructural features of the storage material underlie the nosological spectrum of NCLs $[1,2]$. NCLs are traditionally considered lysosomal storage diseases (LSD), even though several of the mutant proteins are not primary

\footnotetext{
*Correspondence: bbenitez@dom.wustl.edu

'Department of Medicine, Washington University School of Medicine, St. Louis, MO 63110, USA

Full list of author information is available at the end of the article
}

lysosomal proteins. The function of some of these proteins is largely unknown and their relationship to lysosome function is unclear [3].

Adult-onset NCLs (ANCL) represent up to $10 \%$ of NCL cases [4]. ANCL patients exhibit the typical autofluorescent granular osmiophilic deposits (GROD) in neurons and skin cells $[4,5]$. In recent years, the progress in sequencing technology has supported the identification of a plethora of novel disease-causing genes in multiple families with ANCL-like clinical features and GROD-type storage material. This indicates that the genetic architecture of ANCL is more varied and complex than previously thought [3].

Most NCLs have a recessive pattern of inheritance; one exception is the autosomal dominant, adult onset neuronal ceroid lipofuscinosis (AD-ANCL) (MIM \#162350). $\mathrm{AD}-\mathrm{ANCL}$ patients are asymptomatic until around the 
third decade [5]. The first symptom is usually new-onset seizures (tonic-clonic type) followed by rapid cognitive decline, motor impairment and finally, death in the absence of visual impairments [5]. The primary clinical and pathological features of $\mathrm{AD}-\mathrm{ANCL}$ include progressive and widespread neurodegenerative processes resulting in remarkable cortical and cerebellar atrophy [5]. Nearly all of our current understanding of AD-ANCL is based on analysis of postmortem tissue from terminal cases. To our knowledge, there is no report characterizing both early and late pathology events of AD-ANCL cases.

AD-ANCL is caused by a heterozygous point mutation (p.L115R) and an in-frame codon deletion (p.L116 4 ) in the DnaJ (Hsp40) homolog, subfamily $C$, member 5 (DNAJC5) gene [6-8]. The DNAJC5 gene encodes CSP $\alpha$. $C S P \alpha$ is a cytosolic and membrane-bound co-chaperone with a putative neuroprotective function [9]. The function of CSP $\alpha$ in the central nervous system is primarily deduced from data collected from CSP $\alpha$-deficient mice and flies, which exhibit massive age-dependent neurodegeneration affecting mainly active neurons and photoreceptors $[9,10]$. CSP $\alpha$ is widely considered a member of the presynaptic machinery responsible for proteostasis and synapse maintenance [11-13]. Defective synaptic soluble NSF attachment protein receptor (SNARE)complex assembly, due mostly but not exclusively to the reduction in soluble NSF attachment protein-25 (SNAP25) levels, appears to be responsible for the neurodegeneration seen in CSP $\alpha$-deficient mice $[10,11,14,15]$. There are approximately 20 additional proteins that demonstrate altered expression patterns in the absence of CSP $\alpha$ in mice [13] that can also be part of its pathogenic cascade.

The histological hallmark of AD-ANCL is the intracellular accumulation of autofluorescent storage material (AFSM) $[4,5]$. Although there are similarities in the neurodegenerative process between AD-ANCL patients and CSP $\alpha$-deficient mice, no ceroid accumulation has been reported in CSP $\alpha$-deficient mice or flies $[12,16]$. Currently, it is unknown how mutations in DNAJC5/CSP $\alpha$ result in the formation of AFSM. A dominant negative effect was initially proposed as the pathogenic mechanism for AD-ANCL-causing mutations in CSP $\alpha$ based on both its inheritance pattern and a reduction in neuropil CSP $\alpha$ staining in terminal AD-ANCL cases [7, 12, 17].

In this report, we describe the clinical and pathological features of a patient in a clinically early stage of the disease with all the pathological cellular changes found in terminal AD-ANCL cases but with no apparent reduction in CSP $\alpha$ or synaptophysin in the neuropil. In contrast, terminal AD-ANCL patients exhibit significant reductions of presynaptic protein levels, including a significant reduction of CSP $\alpha$.

\section{Materials and methods Mutation analysis}

DNAJC5-Exon4 (Forward, 5'-ggaaggcagtatccccacctggaac-3'; Reverse, 5'-cggcacagtgtcagtgccctcc-3') was amplified on Applied Biosystems (Applied Biosystems, Carlsbad, California, USA) 96-Well GeneAmp ${ }^{\circ}$ PCR System 9700 Thermal Cyclers using a touchdown protocol. PCR products were amplified under the same conditions (25- $\mu$ l volume containing 10x PfuUltra ${ }^{\text {Tx }}$ HF reaction buffer (Stratagene, La Jolla, California, USA), $5 \times$ Betaine (Sigma-aldrich, St Louis, USA), $100 \mu \mathrm{M}$ each dNTP, $200 \mathrm{nM}$ each primer, 0.4 PfuUltra ${ }^{\mathrm{ma}}$ High-Fidelity DNA Polymerase (Promega); PCR profile: $94{ }^{\circ} \mathrm{C}$ followed by 34 cycles of $45 \mathrm{~s}$ at $94{ }^{\circ} \mathrm{C}, 45 \mathrm{~s}$ at $62^{\circ}$, and $1 \mathrm{~min}$ at $72{ }^{\circ} \mathrm{C}$ ). PCR product purification was completed with Exosap-IT (USB Corporation). Sequencing was performed both in forward and reverse direction using BigDye ${ }^{\circ}$ Terminator v3.1 Cycle Sequencing Kit (ABI) on an ABI 3130 sequencer. Sequence traces were analyzed using Sequencher (v4.7, Gene Codes Corp, Ann Arbor, Michigan, USA).

\section{Neuropathological Methods}

After death, the consent for brain removal was obtained from next-of-kin. Briefly, the left hemibrain was preserved in $10 \%$ phosphate-buffered formalin for neuropathologic assessment; the right hemibrain was snap frozen and stored at $-80{ }^{\circ} \mathrm{C}$ for biochemical studies [18]. Unfortunately, no flash frozen tissue was obtained from the early stage AD-ANCL patient. Tissue blocks were taken from the frontal, temporal, parietal, and occipital lobes, amygdala, hippocampus, basal ganglia, thalamus, brainstem, cerebellum, and spinal cord when available. Following formic acid (95\%) pretreatment for $5 \mathrm{~min}$, histology was undertaken and included hematoxylin and eosin and a modified Bielschowsky silver impregnation. Immunohistochemistry was performed using the following primary antibodies: glial fibrillary acidic protein (GFAP, 1:2,000; Dako, Glostrup, Denmark), ionized calcium-binding adapter molecule 1 (Iba1, 1:2,000; Wako Chemicals USA Inc., Richmond, VA), synaptophy$\sin$ (1:1,000; Abcam, Cambridge, MA) and CSP $\alpha$ / DNAJC5 (AB1576, 1:1,000, Millipore, Temecula, CA).

\section{Densitometry}

Densitometry of synaptophysin and CSP $\alpha / D N A J C 5 \mathrm{im}$ munoreactivity was assessed in the cortical ribbon of the middle frontal gyrus, an area available in all mutation carriers and non-carriers, and included the gyral crest and sulcal depth. The intensity of immunoreactivity (luminance measured as arbitrary values) was determined following immunohistochemistry of all cases treated in a single batch using Densita (MicroBrightField, Inc., Williston, VT) software. 


\section{Ultrastructural methods}

Small pieces of tissues from the right palm of formalinfixed tissue were processed for transmission electron microscopy as previously described [5]. Briefly, specimens were fixed overnight in 3 \% glutaraldehyde in Sorensen's buffer, osmicated, dehydrated in graded alcohols and embedded in Epon. Thin sections were cut on an ultramicrotome, collected on mesh grids, stained with lead citrate and uranyl acetate and examined with a JEOL 1200 electron microscope.

\section{Immunoblotting}

Cells were lysed in radioimmune precipitation assay (RIPA) buffer (50 mM Tris- $\mathrm{HCl}, \mathrm{pH} 7.4,150 \mathrm{mM} \mathrm{NaCl}$, $1 \%$ Nonidet P-40 and $0.25 \%$ sodium deoxycholate) plus $1 \mathrm{X}$ phenylmethanesulfonylfluoride (PMSF) and 1X complete protease inhibitor mixture (Sigma, St. Louis, $\mathrm{MO}$ ) for $10 \mathrm{~min}$ on ice and then spun at $14,000 \mathrm{rpm}$ for $10 \mathrm{~min}$ at $4{ }^{\circ} \mathrm{C}$. Protein was subjected to electrophoresis and transferred to PDVF membrane (BIO-RAD, Hercules, CA). The primary antibodies were diluted as follows: CSP $\alpha$ (ADI-VAP-SV003-E; Enzo life science) 1:5000, HSC70/HSP73 (ADI-SPA-816; Enzo life science) 1:1000, Synaptobrevin/VAMP2 (104211, Synaptic System) 1:10000, Syntaxin-1 (110011, Synaptic System) 1:10000, SNAP-25 ( SMI81, Covance) 1:10000, Synaptophysin (ab8049, abcam) 1:5000, Flotillin (C-7, sc-133153, Santa Cruz Biotechnology) 1:5000, $\beta$-Actin (A1978, Sigma-Aldrich) 1:5000. The membranes were then incubated with secondary antibodies, horseradish peroxidase-conjugated anti-mouse or antirabbit IgG (KPL, Gaithersburg, MD) diluted 1:2000 in 4 $\%$ nonfat dry milk PBS-T for $1 \mathrm{~h}$ at room temperature.
Signals were visualized using Lumigen ECL Ultra (TMA-6) (Lumigen, Southfield, MI). Densitometric semi-quantification was performed using Image J software (National Institutes of Health).

\section{Analysis of Clinical Samples}

The Institutional Review Board (IRB) at the Washington University in Saint Louis School of Medicine approved the study. Prior to their participation, written informed consent was reviewed and obtained from family members. The Human Research Protection Office (HRPO) approval number for our ADRC Genetics Core family studies is 201104178. The neuropathological findings of AD-ANCL patients were published previously by Josephson et al. [5], a brief description is found in Table 1. DNAJC5 mutation identification and screening was published by Benitez et al. [6]. The clinically earlystage patient with AD-ANCL belongs to the 7th generation of this family, identified as $(7: 3)$ in the pedigree (Fig. 1) [6].

\section{Statistical analyses}

All data are shown as means \pm SEM. For comparison of two groups, Student's unpaired two-tailed test was used. Data were analyzed using GraphPad Prism, version 5.00 (San Diego, CA).

\section{Case Presentation}

\section{Mutation analyses of DNAJC5/CSPa/NCL4B}

Sequencing analysis of exon 4 of the DNAJC5 gene revealed a coding variant heterozygous transversion change at position c.344 (c.344 T > G) (Fig. 1a) which

Table 1 Demographics of AD-ANCL cases and healthy controls

\begin{tabular}{|c|c|c|c|c|c|c|}
\hline Individual ID & Age at death & Gender & State & Brain wt. (g) & PMI (h) & Cause of death \\
\hline 1 & 37 & $\mathrm{~F}$ & Case & 1160 & 4.5 & Motor vehicle accident \\
\hline 2 & 49 & $\mathrm{~F}$ & Case & 1010 & 13.5 & Bronchopneumonia \\
\hline 3 & 50 & $\mathrm{~F}$ & Case & 1050 & 17 & Bronchopneumonia \\
\hline 4 & 50 & M & Case & 1110 & 22 & Inanition \\
\hline 5 & 53 & F & Case & 1005 & 8 & Parry's disease \\
\hline 6 & 70 & $\mathrm{~F}$ & Control & 1180 & 16 & Carcinoma of colon \\
\hline 7 & 72 & M & Control & 980 & 5 & Respiratory failure \\
\hline 8 & 72 & F & Control & 1060 & 16 & Inanition \\
\hline 9 & 73 & $\mathrm{~F}$ & Control & 1230 & 8 & Carcinoma of lung \\
\hline 10 & 81 & F & Control & 1200 & 21 & Myocardial infarction \\
\hline 11 & 89 & $\mathrm{~F}$ & Control & 1100 & 33 & Respiratory failure \\
\hline 12 & 90 & $\mathrm{~F}$ & Control & 1050 & 22 & Respiratory failure \\
\hline 13 & 90 & $M$ & Control & 1320 & 6.5 & Bronchopneumonia \\
\hline 14 & 91 & $M$ & Control & 1170 & 8.5 & Myocardial infarction \\
\hline 15 & 93 & $\mathrm{~F}$ & Control & 1155 & 5 & Respiratory failure \\
\hline
\end{tabular}

Wt weight, $P M I$ post-mortem interval 


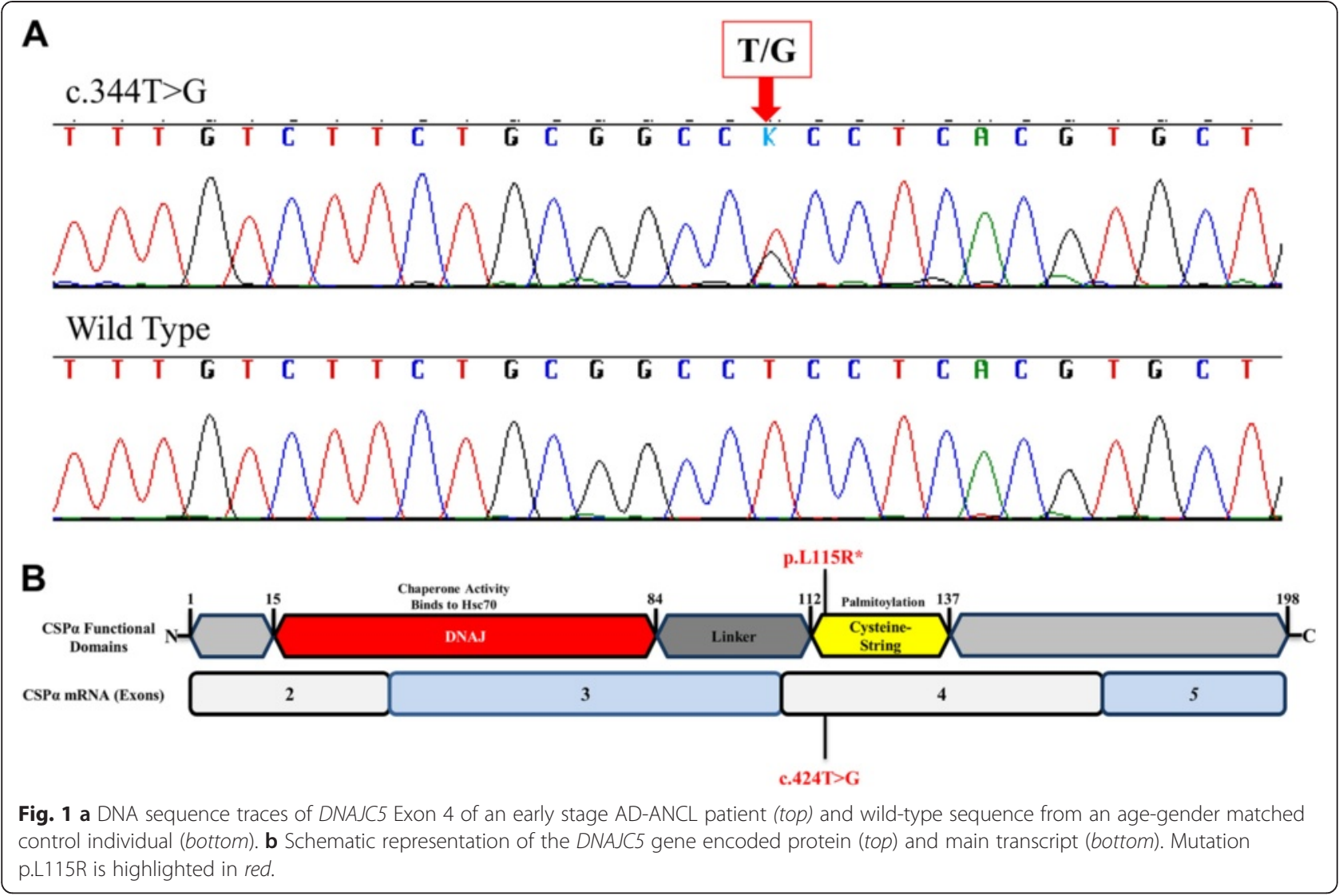

results in a leucine-to-arginine amino acid substitution (Fig. 1b). This mutation was previously found in other members of the same family with terminal ADANCL $[6,7]$.

\section{Clinical features of an early stage AD-ANCL patient}

A member of a multigenerational family with AD-ANCL was evaluated as a research volunteer at age 30 and 33 years at the Knight Alzheimer's Disease Research Center, Washington University, St. Louis, Missouri, as part of the follow-up of family members. At both visits the patient was cognitively normal, had no neurological symptoms, and had an unremarkable neurological examination. Patient's next evaluation was at $36 \mathrm{y}$, when patient reported experiencing multicolored visual hallucinations in the prior 12 months. More recently the patient reported apparent myoclonus affecting the upper extremities and had experienced several non-injurious falls. Three weeks prior to the assessment, patient had an accident while driving her automobile. The patient had no reported seizure episodes. The neurologic examination remained unremarkable and patient again was rated as cognitively normal. An awake electroencephalogram, however, showed bilateral epileptiform discharges. The patient was evaluated seven months later and began medication.

\section{Neuropathology of an early stage AD-ANCL patient}

Neuropathological examination of coronal slices revealed no apparent atrophy of the cortical and subcortical gray and white matter (Fig. 2). There were no changes found in the limbic system or the cerebellum of this patient. There was some depigmentation of the substantia nigra (Fig. 2, inset, right panel), compared to normal control (inset, left panel). We performed histological analysis of the brain and found a marked enlargement of the cortical pyramidal neurons (Fig. 3a). In affected neurons, there is displacement of the nucleus to the base of the apical dendrite and a markedly swollen cell body in comparison to the typical pyramidal shape of neurons in neocortical layer III (Fig. 3b). Neurons contained typical AFSM (Fig. 3c). We also found focal astrogliosis and relatively mild microgliosis adjacent to some swollen neurons in the frontal lobe neocortex (Fig. $3 \mathrm{e}$ and $\mathrm{g}$, respectively).

We also found cuboidal secretory cells containing lipofuscin granules in a palmar sweat gland (Fig. 4a). Electron microscopy revealed cells containing dense and granular osmiophilic lipopigment (Fig. 4b) as well as granular lipopigment and a lipid globule within a cytosomal membrane (Fig. 4c). 


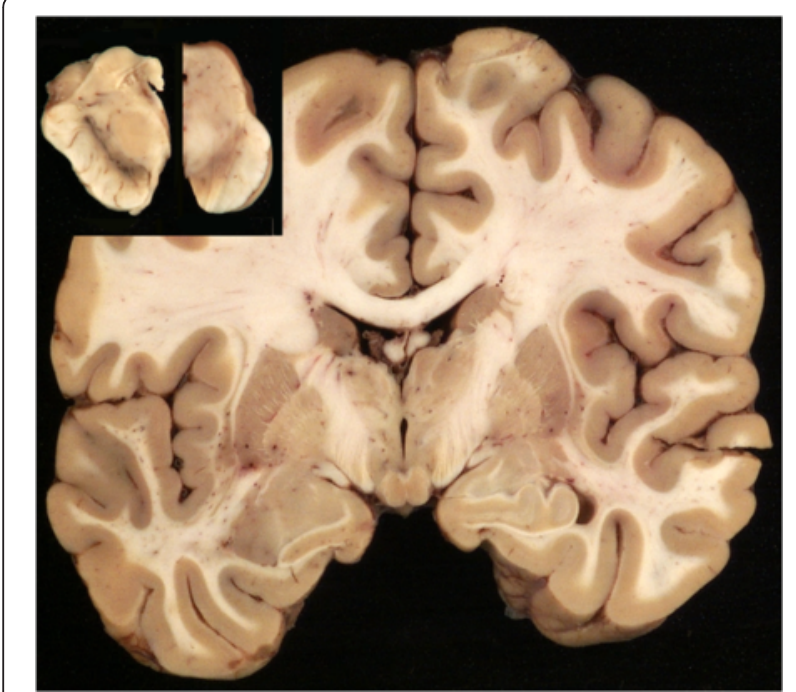

Fig. 2 Macroscopic findings in the brain of an early stage AD-ANCL patient with DNAJC5 p.L115R mutation. The cortical ribbon, basal ganglia, and medial temporal lobe structures at the level of the mammillary bodies are unremarkable. There is some depigmentation of the substantia nigra (Fig. 2, inset, right panel) in comparison with a normal subject (Fig. 2, inset, left panel)
No changes in levels of CSPa or synaptophysin in the brains of an early stage AD-ANCL patient

DNAJC5/CSP $\alpha$ (Fig. 5a, b) and synaptophysin (Fig. 5c, d) immunohistochemistry revealed diffuse neuropil staining of the cerebral cortex, consistent with synaptic localization. Densitometry analysis of the DNAJC5/CSP $\alpha$ and synaptophysin staining was compared with 10 aged non-mutation carrier (NC) controls (for demographic data see Table 1). Although there was a trend towards a decrease in synapse (synaptophysin) density in ADANCL, it was not statistically significant $(\mathrm{p}=0.16$, two tailed t-test). Densitometry of DNAJC5/CSP $\alpha$ immunohistocemistry was also variable, probably due to fixation artifacts, and no difference ( $\mathrm{p}=0.8$, two tailed $\mathrm{t}$-test) in the density of staining between the mutation carriers and non-carriers was observed (Fig. 5f).

\section{Changes in levels of synaptic proteins in the brains of terminal AD-ANCL patients}

We previously predicted that the p.L115R mutation would dramatically decrease the affinity of $\operatorname{CSP} \alpha$ for membranes [6]. Here, we show that mutant CSP $\alpha-$ p.L115R reduces levels of CSP $\alpha$ in the cytosolic fraction by $95 \%(0.05 \pm 0.01$ S.E., $n=6, p=0.001$, unpaired $t$ test) compared to controls, while the membrane-bound fraction (detergent soluble) is reduced by $\sim 50 \%(0.53 \pm$ 0.03 S.E., $\mathrm{n}=6, \mathrm{p}=0.004$, unpaired $\mathrm{t}$-test) in the occipital
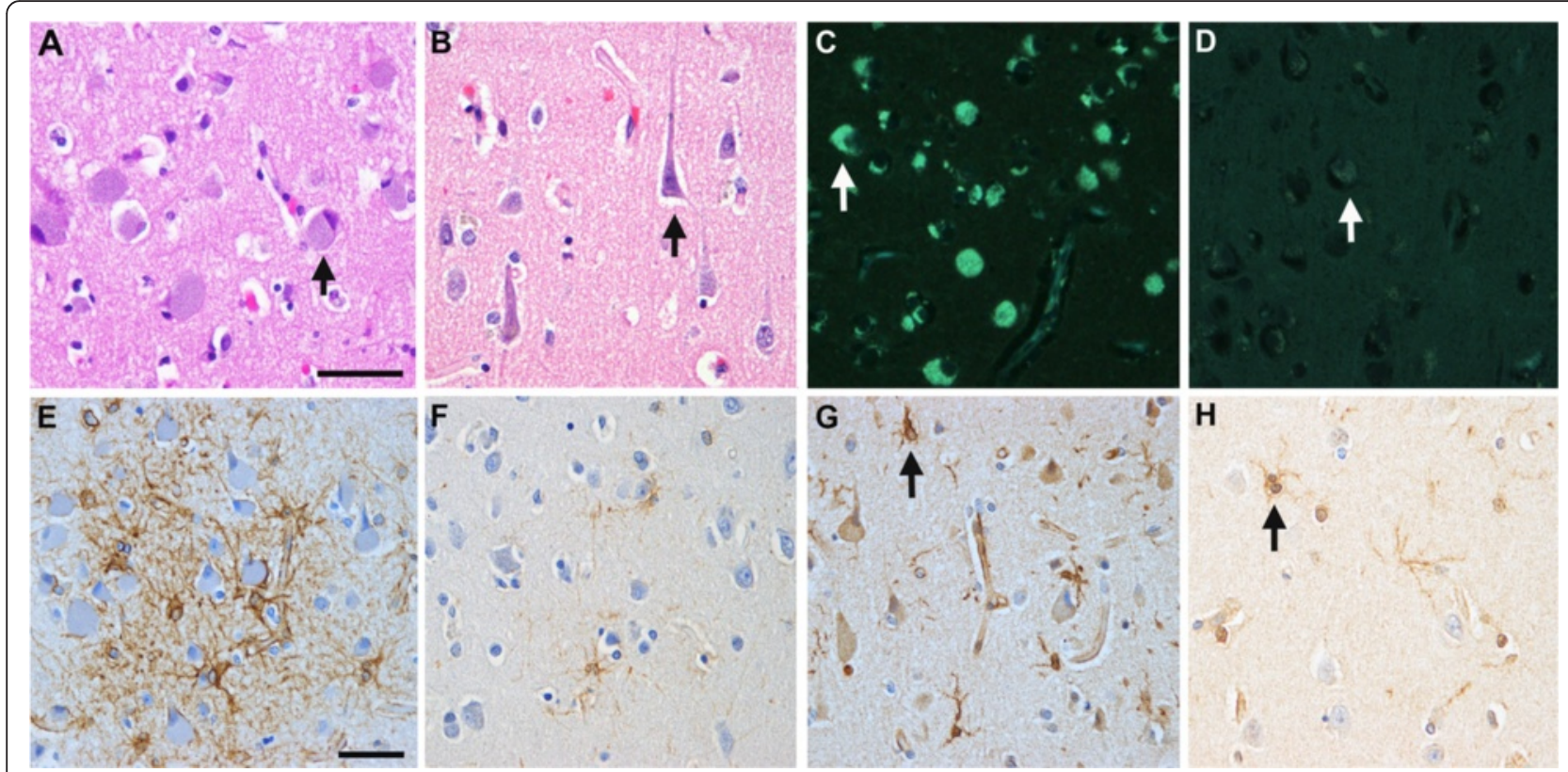

Fig. 3 Microscopy of AD-ANCL patient with DNAJC5 p.L115R mutation. a An enlarged cortical pyramidal neuron with eccentric nucleus in a mutation (MC) (arrow). b A typical pyramidal neuron in a non-mutation carrier (NC) control (arrow). Stain: Hematoxylin and eosin. c An enlarged neuron contains stored lipopigment which is autofluorescent under ultraviolet light (arrow). d NC control exhibits modest neuronal accumulation of lipofuscin (arrow). e, In a MC, there is patchy reactive astrocytosis (GFAP immunohistochemistry) $\mathbf{f}$ Sparse reactive astrocytes in a NC control. $\mathbf{g}$ There is a modest increase in the number of activated microglial cells in the frontal neocortex (arrow) in comparison with a NC control (arrow) (h) (g and $\mathbf{h}$, arrows indicate microglial cells) (Ibal immunohistochemistry). a-d (scale bar in a) bar = $50 \mu \mathrm{m}$; (e-h) (scale bar in e) bar = $50 \mu \mathrm{m}$ 


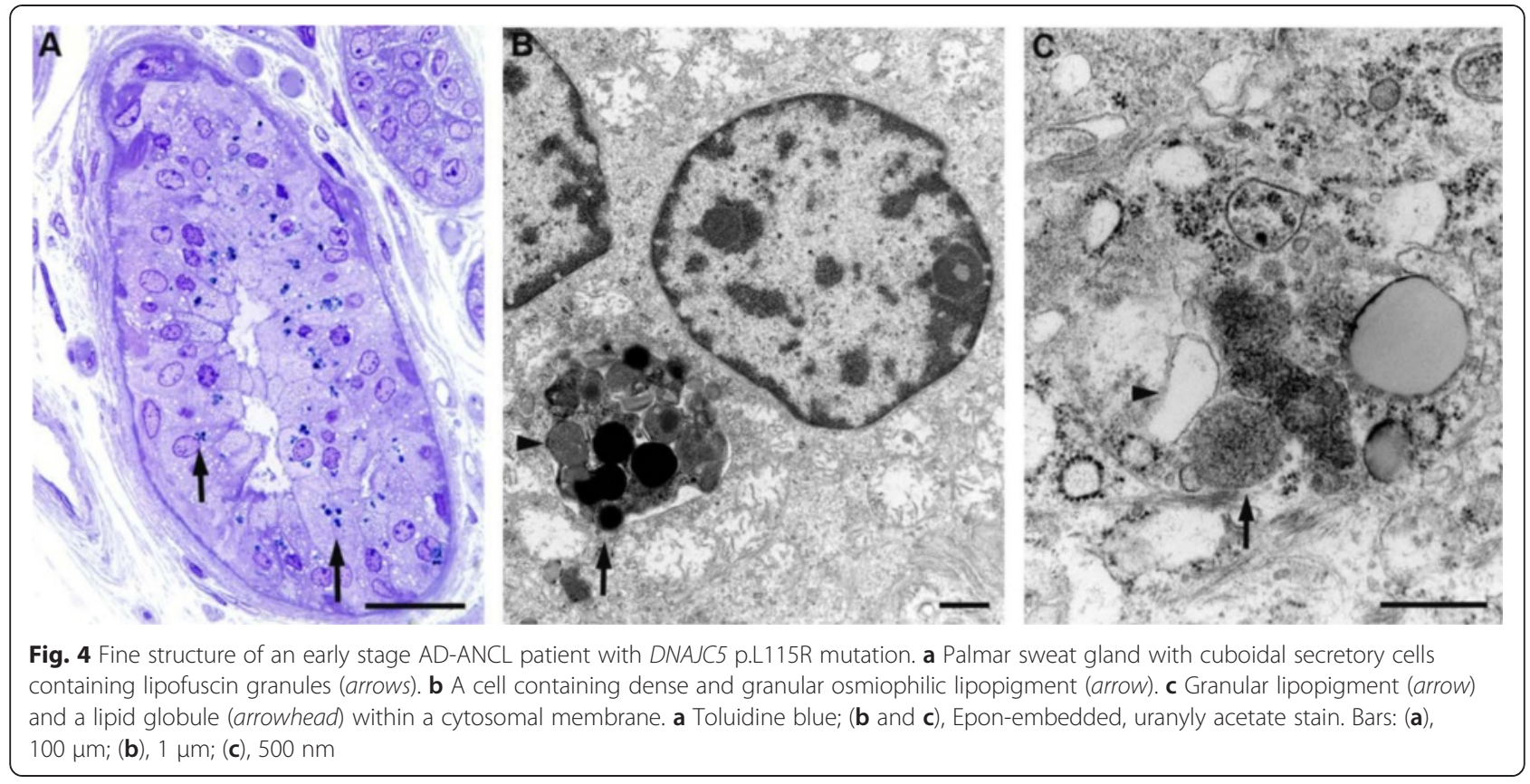

lobe of terminal AD-ANCL patients compared to controls (Fig. 6a). Detailed analysis of CSP $\alpha$-deficient mice has shown that neurodegeneration in the absence of $C S P \alpha$ is a consequence of the defective function of SNAP-25 and SNARE binding proteins [14]. Therefore, we examined whether the CSP $\alpha$ reduction found in terminal AD-ANCL patients was accompanied by changes in other synaptic proteins. We found a significant reduction in several SNARE-complex forming presynaptic proteins including, but not limited to a $59 \%$ reduction in SNAP-25 levels, $41 \%$ reduction in synaptobrevin/ VAMP2 levels and a $43 \%$ reduction in syntaxin 1 levels in brains of terminal AD-ANCL patients compared to controls. We also found a $36 \%$ reduction in synaptophysin levels and no changes in CSP 's partner, HSC7O levels (Fig. 6a). The reduction of the presynaptic proteins was similar across the different brain regions (frontal, parietal and temporal) or cerebellum among the ADANCL patients (Data not shown). No flash frozen tissue was obtained from the early stage AD-ANCL patient, which prevents us from performing the same biochemical analyses.

\section{Discussion}

AD-ANCL is a rare multisystem neurodegenerative disorder characterized by intracellular accumulation of macromolecular debris $[4,5]$, caused by p.L115R (Fig. 1) and p.L116 $\triangle$ mutations in DNAJC5/CLN4B/CSPa $[6,7$, 8]. Nearly all of our understanding of AD-ANCL is based on analysis of postmortem tissue from terminal cases [5, 7]. Here, we describe a 37 year old patient in a clinically early stage of AD-ANCL harboring a p.L115R mutation in $D N A J C 5 / C S P \alpha$. This patient was a member of a multigenerational family with AD-ANCL, which has been clinically and pathologically described by Josephson et al. [5]. The average age at onset in the other members of this family is $36 \pm 2.44$ years (range 32-40) with a duration of $9.3 \pm$ 3.3 years (range $5-13$ ). The clinical symptoms start with new-onset generalized tonic-clonic seizures followed by dementia (aprox. 3 years), motor impairment and finally death [5]. Interestingly, most of the histopathological findings found in this patient are indistinguishable from the changes reported in terminal cases, including the clear presence of AFSM in the soma of neurons and most prominent in pyramidal neurons (Fig. 3c) [5]. However, unlike the terminal cases, there was no brain atrophy or significant differences in CSP $\alpha$ or synaptophysin in the neuropil compared to the controls (Fig. 5). The effect of these mutations on CSP $\alpha$, a synaptic protein, and how this results in AD-ANCL has not been established. This study demonstrates alterations in the neuronal soma, most likely in the lysosome, of an early-stage of the disease (Fig. 3a-d). In contrast, massive synaptic degeneration was only observed in post-mortem tissue from terminal AD-ANCL patients (Fig. 6a, b) [5, 7], suggesting that the dysfunction in the soma of neurons likely at the lysosomal level occurs before the massive synaptic degeneration as a consequence of the aggregatederived toxic effects of the mutation in DNAJC5/ $\operatorname{CSP} \alpha[16]$.

Neuronal cell death seems to be correlated with age of onset and duration of the disease in most NCLs [19]. In 


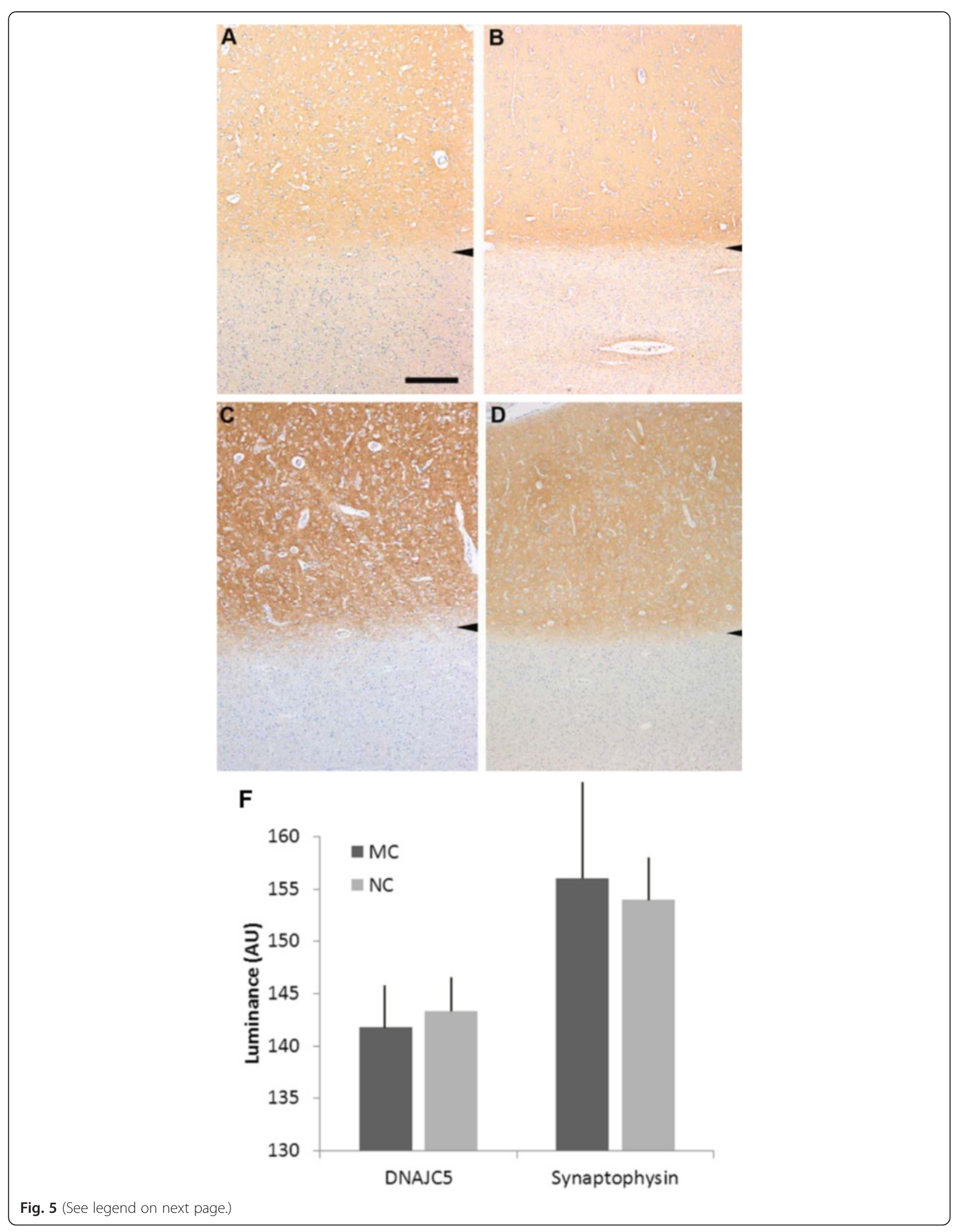


(See figure on previous page.)

Fig. 5 Immunohistochemistry for CSPa and synaptophysin in the cerebral cortex of an early stage AD-ANCL patient (a) DNAJC5/CSPa immunohistochemistry in the cerebral cortex of an early stage AD-ANCL patient (zone of staining above arrowhead); the underlying white matter is unstained. b non-mutation control subject. c Immunohistochemistry for synaptophysin in the cerebral cortex of an early stage AD-ANCL patient (zone of staining above arrowhead); the underlying white matter is unstained. $\mathbf{d}$ non-mutation control subject. Bar: 500 um. $\mathbf{f}$ Quantification of the intensity of immunoreactivity staining (luminance measured as arbitrary values) (graph). Values represent the mean \pm S.E.M. of three independent experiments using Student's $t$ test

AD-ANCL there is a remarkable neuronal depletion in the cortex of the frontal, parietal and temporal lobes of the AD-ANCL terminal cases [5]. Here, we found minimal to no evidence of neuronal loss or cortex atrophy in a clinically early-stage patient with AD-ANCL in the examined areas.

Microgliosis and astrogliosis seems to be an early event, possibly even occurring before neuronal degeneration in several NLCs [19, 20] including adult forms [5], and remains remarkable in very advanced stages of neurodegeneration in the cerebral cortex $[5,19]$. Here, we found microglial and astroglial activation in isolated areas surrounding the swollen neurons in the cortex of an early-stage AD-ANCL patient.

Here, we report that terminal AD-ANCL patients exhibit reductions in presynaptic proteins that form the
SNARE complex including SNAP-25, VAMP2 and STX1 (Fig. 6a, b). A similar reduction in SNAP-25 was reported in CSP $\alpha$-deficient mice $[9,10,14]$ but unlike the findings in CSP $\alpha$-deficient mice, terminal AD-ANCL patients exhibit similar HSC70 levels compared to control (Fig. 5b). Other LSDs also exhibit SNARE defects as a consequence of the primary lysosomal defects [21]. Unfortunately, lysosome function has not been evaluated in CSP $\alpha$-deficient mice $[12,16]$. In contrast, CSP $\alpha$-p.L115R induces dramatic changes in the soma that appear to precede the synaptic defects. This sequence of events has been reported in other LSDs where non-lysosomal mutant proteins affect the intracellular trafficking of proteins upstream or downstream of the lysosome, including synaptic proteins, subsequently producing synaptic dysfunction [22-24].
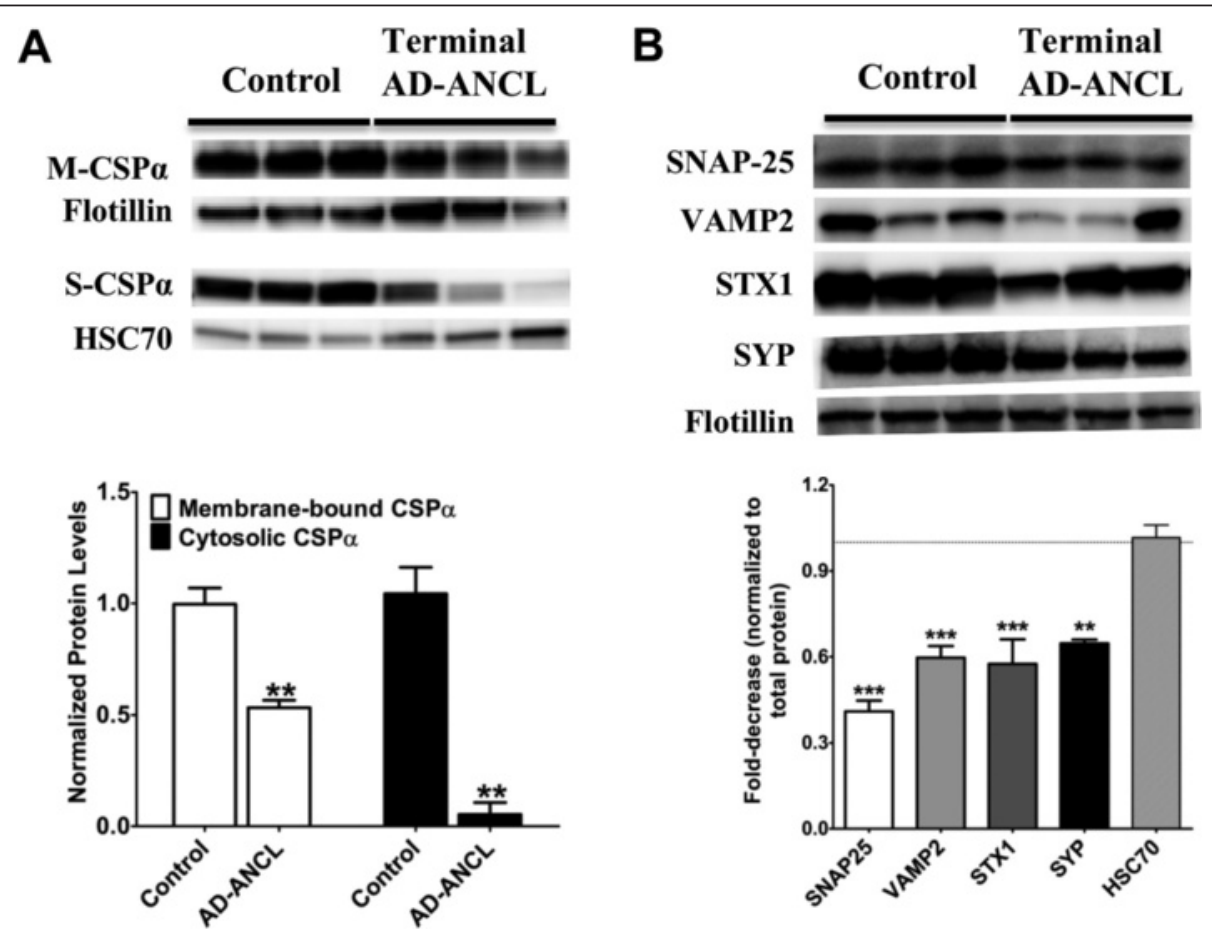

Fig. 6 Presynaptic protein levels in the brains of terminal AD-ANCL patients. a Representative western blots (top) and semi-quantitative analysis (graph, bottom) displaying the protein levels of CSPa in the membrane (M-CSPa) (normalized to Flotillin) and cytosolic/soluble (S-CSPa) (normalized to HSC70) fractions from the occipital lobe of three controls and three terminal AD-ANCL patients. The graphs show the expression level of the indicated proteins normalized to flotillin or HSC70 expression. b Representative western blots (top) and semi-quantitative analysis (graph, bottom) show the protein levels of SNAP-25, vesicle-associated membrane protein 2 (VAMP2/ Synaptobrevin), Syntaxin 1 (STX1), and Synaptophysin (SYP) in the occipital lobe from three controls and three terminal AD-ANCL patients in the membrane fraction. Values represent the mean \pm S.E.M. of three independent experiments. ${ }^{* *}, p \leq 0.01 ;{ }^{* *}, p \leq 0.001$ using Student's $t$ test 
We have confirmed and extended the finding that postmortem brain samples from terminal AD-ANCL patients display a large reduction in $C S P \alpha$ levels compared to controls (Fig. 6a) [7, 17, 25]. Interestingly, the levels of membrane-bound $\operatorname{CSP} \alpha$ are decreased $50 \%$ in terminal AD-ANCL patients (Fig. 6a). CSP $\alpha$ hemizygous mice exhibit a $50 \%$ reduction in the level of $\operatorname{CSP} \alpha$ and are phenotypically normal [10]. Assuming membrane-bound CSP $\alpha$ is the main functional fraction, this 50 \% reduction alone would not explain the synaptic changes and neurodegeneration found in terminal AD-ANCL patients (Fig. 6a, b). This suggests that an additional mechanism other than an isolated reduction in CSP $\alpha$ levels is responsible for the neurodegeneration found in AD-ANCL. However, we cannot rule out the possibility that the overall reduction, up to one third of the control levels, in both soluble and membrane-bound CSP $\alpha$ found in AD-ANCL patients could be responsible for the synaptic defects seen.

We also found in brain homogenates from individual controls that there is a substantial amount of CSP $\alpha$ present in the soluble/cytosolic fraction (Fig. 6a). CSP $\alpha$ levels in this particular fraction are dramatically reduced in AD-ANCL patients (Fig. 6a). The cytosolic CSP $\alpha$ fraction extracts from the rat brain have been described to interact with $H S C 70$ [26]. The amount of soluble/cytosolic $\operatorname{CSP} \alpha$ depends on both the cell type [27-29] and the activity of palmitoyl- transferases on CSP $\alpha$ [30]. Thus, it is possible that human brain expression levels and types of palmitoyl- transferases vary in different cell types [31] or that palmitoyl- transferase activity is age-dependent resulting in a greater soluble/cytosolic CSP $\alpha$ fraction. We cannot rule out the possibility that this finding is an artifact or contamination from small vesicles in our detergent-free fraction. However, the CSP $\alpha$ levels found in three different individual controls strongly suggest that this is not an artifact (Fig. 6a). The role of soluble/cytosolic CSP $\alpha$ is not clearly defined since the most thoroughly studied is membrane-bound CSP $\alpha$. However, CSP $\alpha$ exhibits both chaperone and co-chaperone activity that does not depend on its ability to bind the membrane.

The understanding of how mutations in a "synaptic" protein can lead to lysosome dysfunction is currently unclear. Lysosomes are dynamic organelles that receive and degrade macromolecules from secretory, endocytic, autophagic and phagocytic membrane-trafficking pathways [32]. Lysosomes also serve as a common endpoint for multiple vesicle-based trafficking systems [32]. CSP $\alpha$ is critical for maintaining vesicle-membrane fusion events [28]. The fusion events between lysosomes and endosomes, phagosomes, autophagosomes or plasma membrane, are all mediated by the SNARE complexes [32]. The main proteins forming these trans-SNARE complexes include syntaxin-7, syntaxin-8 (Q-SNARES) and vesicleassociated membrane protein-8 (VAMP8) and VAMP7
(R-SNARES), in addition to synaptosome-associated protein of $23 \mathrm{kDa}$ (SNAP23), synaptotagmin-VII and Rab3a [32], some of which have been identified as CSP $\alpha$ 's partners [16]. Terminal AD-ANCL patients exhibit significant reductions in SNARE-complex forming proteins (Fig. 6b). Thus, it is likely that dysfunctional CSP $\alpha$ affects the machinery that coordinates these lysosomal fusion events resulting in the formation of the ceroid aggregates and subsequent swelling of the cellular soma (cortical pyramidal neurons and palmar sweat gland) seen in AD-ANCL.

In this report, we describe the clinical and pathological features of a patient in a clinically early stage of ADANCL with all the pathological cellular changes found in terminal AD-ANCL cases, but with minimal or no apparent reduction in CSP $\alpha$ or synaptophysin in the neuropil. In contrast, terminal AD-ANCL patients exhibit significant reductions of presynaptic protein levels, including a significant reduction in $C S P \alpha$ and its main partner SNAP25 but not in HSC7O levels. Our analysis of a single brain region in an early-stage AD-ANCL patient showed a nonsignificant trend toward synaptic loss (Fig. 5a). CSP $\alpha$-deficient mice exhibit a massive neurodegeneration with wide heterogeneity in the time-course and type of neurons affected [10]. Thus, it seems possible that this early-stage AD-ANCL patient might have synaptic losses that are not obvious in gross immunohistological analyses.

In summary, the widespread neurodegeneration seen in terminal AD-ANCL patients seems to be a consequence of both the early effects of mutant CSP $\alpha$ in the soma, most likely on lysosome function, and subsequent synaptic dysfunction resulting from the reduction in CSP $\alpha$ levels. We hypothesize that in this AD-ANCL patient the lysosomal dysfunction (ceroid accumulation) occurred before neuronal cell death and synaptic degeneration.

\section{Competing interest}

The authors declare that they have no conflicts of interest with the contents of this article.

\section{Authors' contributions}

Conception and design of the study was by BAB and MSS; Acquisition and analysis of clinical data wasby JCM and JBN; Acquisition of genetic and biochemical data was by BAB; Analysis of genetic datawas by BAB and CC; Acquisition and analysis of neuropathological data was by NJC; Acquisition and analysis of electron microscopy data was by RES The manuscript was written by BAB and MSS.

\section{Acknowledgments}

The authors especially want to thank the patients and their families, whose help and participation made this work possible. This work was funded in part by grants from the National Institutes of Health (NS043205 and NS084861 to MSS; P50 AG005681 and P01 AG003991 to JCM/NJC) and financial support from the Frye Family Foundation to MSS. This work was also supported by 2014 pilot funding from the Hope Center for Neurological Disorders and the Danforth Foundation Challenge at Washington University to BAB and CC. We thank Dr. Shonali Midha who provided editing and insightful discussion of the manuscript. 


\section{Author details}

'Department of Medicine, Washington University School of Medicine, St. Louis, MO 63110, USA. ²Department of Neurology, Washington University School of Medicine, St. Louis, MO 63110, USA. ${ }^{3}$ Department of Pathology \& Immunology, Washington University School of Medicine, St. Louis, MO 63110, USA. ${ }^{4}$ Department of Psychiatry, Washington University School of Medicine, St. Louis, MO 63110, USA. ${ }^{5}$ Department of Genetics, Washington University School of Medicine, St. Louis, MO 63110, USA. ${ }^{6}$ Hope Center for Neurological Disorders, Washington University School of Medicine, St. Louis, MO 63110, USA.

Received: 28 September 2015 Accepted: 13 November 2015

Published online: 26 November 2015

\section{References}

1. Haltia M, Goebel HH. The neuronal ceroid-lipofuscinoses: A historical introduction. Biochim Biophys Acta - Mol Basis Dis. 2013;1832:1795-800. doi:10.1016/j.bbadis.2012.08.012

2. Anderson GW, Goebel HH, Simonati A. Human pathology in NCL. Biochim Biophys Acta - Mol Basis Dis. 2013;1832:1807-26. doi:10.1016/j.bbadis.2012.11.014.

3. Cotman SL, Karaa A, Staropoli JF, Sims KB. Neuronal ceroid lipofuscinosis: Impact of recent genetic advances and expansion of the clinicopathologic spectrum topical collection on genetics. Curr Neurol Neurosci Rep. 2013. doi:10.1007/s11910-013-0366-Z.

4. Sadzot B, Reznik M, Arrese-Estrada JE, Franck G. Familial Kufs' disease presenting as a progressive myoclonic epilepsy. J Neurol. 2000;247:447-54.

5. Josephson SA, Schmidt RE, Millsap P, McManus DQ, Morris JC. Autosomal dominant Kufs' disease: a cause of early onset dementia. J Neurol Sci. 2001; 188:51-60.

6. Benitez BA, Alvarado D, Cai Y, Mayo K, Chakraverty S, Norton J, et al. Exomesequencing confirms DNAJC5 mutations as cause of adult neuronal ceroidlipofuscinosis. PLoS One. 2011;6:e26741. doi:10.1371/journal.pone.0026741.

7. Noskova L, Stranecky V, Hartmannova H, Pristoupilova A, Baresova V, Ivanek $\mathrm{R}$, et al. Mutations in DNAJC5, encoding cysteine-string protein alpha, cause autosomal-dominant adult-onset neuronal ceroid lipofuscinosis. Am J Hum Genet. 2011;89:241-52. doi:10.1016/j.ajhg.2011.07.003.

8. Velinov M, Dolzhanskaya N, Gonzalez M, Powell E, Konidari I, Hulme W, et al. Mutations in the gene DNAJC5 cause autosomal dominant Kufs disease in a proportion of cases: study of the Parry family and 8 other families. PLoS One. 2012;7, e29729. doi:10.1371/journal.pone.0029729.

9. Zinsmaier KE. Cysteine-string protein's neuroprotective role. J Neurogenet. 2010;24:120-32. doi:10.3109/01677063.2010.489625.

10. Fernandez-Chacon R, Wolfel M, Nishimune H, Tabares L, Schmitz F, Castellano-Munoz M, et al. The synaptic vesicle protein CSP alpha prevents presynaptic degeneration. Neuron. 2004;42:237-51.

11. Chandra S, Gallardo G, Fernandez-Chacon R, Schluter OM, Sudhof TC. Alphasynuclein cooperates with CSPalpha in preventing neurodegeneration. Cell. 2005;123:383-96. doi:10.1016/j.cell.2005.09.028.

12. Donnelier J, Braun JE. CSPalpha-chaperoning presynaptic proteins. Front Cell Neurosci. 2014;8:116. doi:10.3389/fncel.2014.00116.

13. Zhang $Y Q$, Henderson MX, Colangelo CM, Ginsberg SD, Bruce C, Wu T, et al. Identification of CSPalpha clients reveals a role in dynamin 1 regulation. Neuron. 2012;74:136-50. doi:10.1016/j.neuron.2012.01.029.

14. Sharma M, Burre J, Bronk P, Zhang Y, Xu W, Sudhof TC. CSPalpha knockout causes neurodegeneration by impairing SNAP-25 function. EMBO J. 2012;31:829-41. doi:10.1038/emboj.2011.467.

15. Sharma M, Burre J, Sudhof TC. CSPalpha promotes SNARE-complex assembly by chaperoning SNAP-25 during synaptic activity. Nat Cell Biol. 2011;13:30-9. doi:10.1038/ncb2131.

16. Burgoyne RD, Morgan A. Cysteine string protein (CSP) and its role in preventing neurodegeneration. Semin Cell Dev Biol. 2015. doi:10.1016/j. semcdb.2015.03.008

17. Donnelier J, Braun ST, Dolzhanskaya N, Ahrendt E, Braun AP, Velinov M, et al. Increased Expression of the Large Conductance, Calcium-Activated K+ (BK) Channel in Adult-Onset Neuronal Ceroid Lipofuscinosis. PLoS One. 2015;10:e0125205. doi:10.1371/journal.pone.0125205.

18. Cairns NJ, Perrin RJ, Franklin EE, Carter D, Vincent B, Xie M, et al. Neuropathologic assessment of participants in two multi-center longitudinal observational studies: the Alzheimer Disease Neuroimaging Initiative (ADNI) and the Dominantly Inherited Alzheimer Network (DIAN). Neuropathology. 2015;35:390-400. doi:10.1111/neup.12205.
19. Williams RE, Aberg L, Autti T, Goebel HH, Kohlschütter A, Lönnqvist T. Diagnosis of the neuronal ceroid lipofuscinoses: An update. Biochim Biophys Acta - Mol Basis Dis. 2006;1762:865-72. doi:10.1016/j.bbadis.2006.07.001.

20. Shyng C, Sands MS. Astrocytosis in infantile neuronal ceroid lipofuscinosis: friend or foe? Biochem Soc Trans. 2014;42:1282-5. doi:10.1042/BST20140188.

21. Fraldi A, Annunziata F, Lombardi A, Kaiser HJ, Medina DL, Spampanato C, et al. Lysosomal fusion and SNARE function are impaired by cholesterol accumulation in lysosomal storage disorders. EMBO J. 2010;29:3607-20. doi:10.1038/emboj.2010.237.

22. Kielar C, Wishart TM, Palmer A, Dihanich S, Wong AM, Macauley SL, et al. Molecular correlates of axonal and synaptic pathology in mouse models of Batten disease. Hum Mol Genet. 2009;18:4066-80. doi:10.1093/hmg/ddp355.

23. Pressey SN, Smith DA, Wong AM, Platt FM, Cooper JD. Early glial activation, synaptic changes and axonal pathology in the thalamocortical system of Niemann-Pick type C1 mice. Neurobiol Dis. 2012;45:1086-100. doi:10.1016/j. nbd.2011.12.027

24. Song JW, Misgeld T, Kang H, Knecht S, Lu J, Cao Y, et al. Lysosomal activity associated with developmental axon pruning. J Neurosci. 2008;28:8993-9001. doi:10.1523/JNEUROSCI.0720-08.2008.

25. Greaves J, Lemonidis K, Gorleku OA, Cruchaga C, Grefen C, Chamberlain LH. Palmitoylation-induced aggregation of cysteine-string protein mutants that cause neuronal ceroid lipofuscinosis. J Biol Chem. 2012;287:37330-9. doi:10.1074/jbc.M112.389098.

26. Evans GJ, Morgan A. Phosphorylation-dependent interaction of the synaptic vesicle proteins cysteine string protein and synaptotagmin I. Biochem J. 2002;364:343-7. doi:10.1042/BJ20020123.

27. Brown $H$, Larsson O, Branstrom R, Yang SN, Leibiger B, Leibiger I, et al. Cysteine string protein (CSP) is an insulin secretory granule-associated protein regulating beta-cell exocytosis. EMBO J. 1998;17:5048-58. doi:10.1093/emboj/17.17.5048.

28. Chamberlain LH, Burgoyne RD. Cysteine string protein functions directly in regulated exocytosis. Mol Biol Cell. 1998;9:2259-67.

29. Chamberlain LH, Henry J, Burgoyne RD. Cysteine string proteins are associated with chromaffin granules. J Biol Chem. 1996;271:19514-7.

30. Greaves J, Salaun C, Fukata Y, Fukata M, Chamberlain LH. Palmitoylation and membrane interactions of the neuroprotective chaperone cysteine-string protein. J Biol Chem. 2008;283:25014-26. doi:10.1074/jbc.M802140200.

31. Greaves J, Chamberlain LH. DHHC palmitoyl transferases: substrate interactions and (patho)physiology. Trends Biochem Sci. 2011;36:245-53. doi:10.1016/j.tibs.2011.01.003.

32. Luzio JP, Pryor PR, Bright NA. Lysosomes: fusion and function. Nat Rev Mol Cell Biol. 2007:8:622-32. doi:10.1038/nrm2217.

\section{Submit your next manuscript to BioMed Central and we will help you at every step:}

- We accept pre-submission inquiries

- Our selector tool helps you to find the most relevant journal

- We provide round the clock customer support

- Convenient online submission

- Thorough peer review

- Inclusion in PubMed and all major indexing services

- Maximum visibility for your research

Submit your manuscript at www.biomedcentral.com/submit 